\title{
Photophysics of indole upon x-ray absorption
}

Thomas Kierspel, ${ }^{a, b, c}$ Cédric Bomme, ${ }^{d}$ Michele Di Fraia, ${ }^{a, b, e}$ Joss Wiese, ${ }^{a, f}$ Denis Anielski, ${ }^{d}$ Sadia Bari, ${ }^{d, g}$ Rebecca Boll, ${ }^{d, g}$ Benjamin Erk, ${ }^{d}$ Jens S. Kienitz, ${ }^{a, b, c}$ Nele L. M. Müller, ${ }^{a, d}$ Daniel Rolles, ${ }^{d, h}$ Jens Viefhaus, ${ }^{d}$ Sebastian Trippel, ${ }^{a, b *}$ and Jochen Küpper ${ }^{a, b, c, f}$

A photofragmentation study of gas-phase indole $\left(\mathrm{C}_{8} \mathrm{H}_{7} \mathrm{~N}\right)$ upon single-photon ionization at a photon energy of $420 \mathrm{eV}$ is presented. Indole was primarily inner-shell ionized at its nitrogen and carbon $1 s$ orbitals. Electrons and ions were measured in coincidence by means of velocity map imaging. The angular relationship between ionic fragments is discussed along with the possibility to use the angle-resolved coincidence detection to perform experiments on molecules that are strongly oriented in their recoil-frame. The coincident measurement of electrons and ions revealed fragmentation-pathway-dependent electron spectra, linking the structural fragmentation dynamics to different electronic excitations. Evidence for photoelectron-impact self-ionization was observed.

David W. Pratt originally initiated our investigations into the photophysics of indole and this paper is dedicated to him on the occasion of his $80^{\text {th }}$ birthday.

July 20, 2018

Keywords: indole, photophysics, fragmentation, x-ray, PEPIPICO

\section{Introduction}

Indole, the chromophore of the essential amino acid tryptophan, is an ubiquitous part of peptides and proteins. It is the strongest near ultraviolet (UV) absorber in these biological molecules and, for a detailed understanding of the photostability and radiation damage of these biological samples, it is highly relevant to disentangle indole's intrinsic photophysics, e. g., its various excitation, relaxation, and fragmentation pathways following electronic excitation. Indole was extensively studied using microwave ${ }^{[12]}$ and optical spectroscopy, $[3,10]$ including vibrationally [910] and rotationally resolved $[3,8]$ electronic spectroscopy, and also using time-resolved ion and photoelectron spectroscopy. ${ }^{[1]-13]}$ Here, we extend these studies to the investigation of the photophysics and photofragmentation dynamics of indole following soft x-ray absorption.

Fragmentation studies of isolated gas-phase molecules and clusters allow to extract molecular properties, such as the geometric structure. 14 15] Therefore, they provide a link between the laboratory frame and the molecular frame that allows to investigate wave packet dynamics on complex potential energy surfaces through molecular-frame dependent observables such as, for instance, molecular-frame angle-resolved photoelectron spectroscopy (MF-ARPES). ${ }^{[16 \mid 17]}$ Furthermore, fundamental relaxation processes like Auger decay, interatomic (intermolecular) Coulombic decay, 18 or electron-transfer mediated decay (ETMD) [20] can be investigated upon x-ray ionization, and can be employed as observables to study molecular dynamics. In order to understand the complete fragmentation and charge rearrangement dynamics of molecules and small compound sys-

a Center for Free-Electron Laser Science, Deutsches Elektronen-Synchrotron DESY, 22607 Hamburg, Germany

${ }^{b}$ Center for Ultrafast Imaging, Universität Hamburg, 22761 Hamburg, Germany

${ }^{c}$ Department of Physics, Universität Hamburg, 22761 Hamburg, Germany

${ }^{d}$ Deutsches Elektronen-Synchrotron DESY, 22607 Hamburg, Germany

${ }^{e}$ Elettra-Sincrotrone Trieste S.C.p.A., 34149, Basovizza, Italy

${ }^{f}$ Department of Chemistry, Universität Hamburg, 20146 Hamburg, Germany

${ }^{g}$ European XFEL GmbH, 22869 Schenefeld, Germany

${ }^{h}$ J.R. Macdonald Laboratory, Department of Physics, Kansas State University, Man-

hattan, KS 66506, USA

*sebastian.trippel@cfel.de; https://www.controlled-molecule-imaging.org tems such as clusters, coincidence measurements can be highly advantageous. [21] Various techniques were developed during the last years, 22 which include photoion-photoion coincidence (PIPICO), photoelectron-photoion-photoion coincidence (PEPIPICO), or Auger-electron photoion-photoion coincidence (AEPIPICO) measurements. 24] Such coincidence measurements can, at least for simple molecules, be used to study molecular-frame (MF) properties by reconstructing the molecular orientation from the measured three-dimensional (3D) velocity distributions of all charged fragments, which is the recoilframe (RF) of the molecule. The connection between the RF and the MF requires unique molecular fragments, e. g., "marker atoms", and prior knowledge about the directionality of the fragmentation to determine the orientation of the molecule within the RF. Studies in the RF include recoil-frame angle-resolved photoelectron spectra (RF-ARPES), 29]34 38 which allow to image molecular orbitals and their temporal evolution during dis-

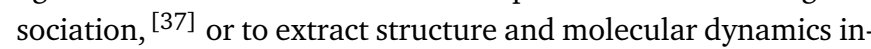
formation by "diffraction from within" 39 type of experiments. For such experiments, it is highly advantageous to locally ionize the molecule at a specific atom, which can be achieved by innershell ionization via extreme ultraviolet radiation, soft $\mathrm{x}$-ray, or $\mathrm{x}$-ray radiation. Localized ionization provides also access to the local electronic structure and excited state dynamics, 37/40]41] and can be used to break specific bonds. 4 [42]

Here, isolated indole $\left(\mathrm{C}_{8} \mathrm{H}_{7} \mathrm{~N}\right)$ molecules were ionized by a single (soft) x-ray photon with an energy of $420 \mathrm{eV}$, i. e., $\sim 10 \mathrm{eV}$ above the nitrogen $1 s$ ionization threshold, the $\mathrm{N}(1 s)$ edge. This gives rise to an enhanced localized ionization at the nitrogen atom in the molecule Photo- and Auger elec-

\footnotetext{
$\dagger$ At a photon energy of $420 \mathrm{eV}$, the nitrogen atom has the highest atomic cross section $\left(0.6466 \cdot 10^{-22} \mathrm{~m}^{2}\right)$ of the molecule's constituents, followed by carbon atoms $\left.\left(0.4327 \cdot 10^{-22} \mathrm{~m}^{2}\right) .43\right]$ In total, the indole monomer contains eight carbon and one nitrogen atom, leading to a probability of $16 \%$ that the complex is locally ionized out of the the nitrogen $1 \mathrm{~s}$ orbital, assuming that the molecular cross sections for the $1 s$ orbitals do not differ significantly from the atomic ones, and neglecting the contribution from the inner-valence and valence orbitals, which are estimated to be on the order of a few percent. The photoabsorption cross section for atomic hydrogen is 3000 times smaller than for nitrogen and is not taken into account.
} 


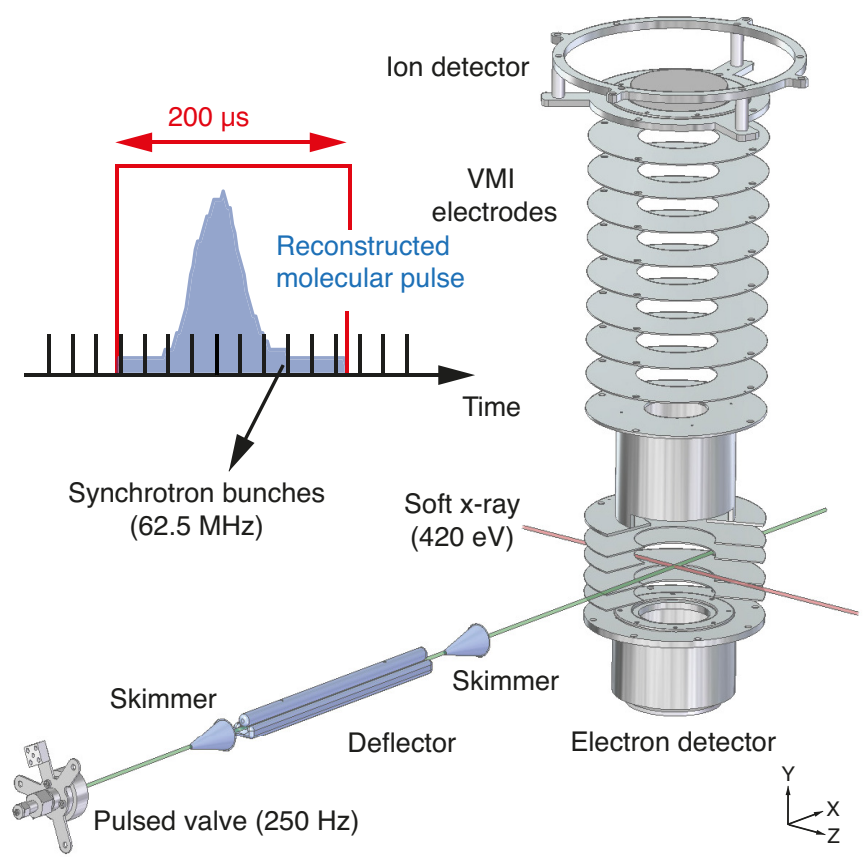

Fig. 1 Experimental setup showing the pulsed valve, skimmers, deflector, the double-sided VMIS, and the synchrotron beam, which crosses the molecular beam in the center of the VMIS. 50 A reconstructed molecular pulse is shown in the top left part. Schematically indicated is the logical gate (red) synchronized to the molecular beam. Multiple synchrotron pulses (black vertical bars) are crossing the molecular beam. Due to the low interaction probability of the synchrotron pulses with the molecular beam and background gas, only a few events per molecular beam pulse were detected.

trons as well as the ionic fragments of indole were detected in coincidence in a double-sided velocity map imaging (VMI) spectrometer (VMIS). 44] Our work provides the first innershell photoionization study of bare gas-phase indole. It also provides the basis for relaxation and fragmentation studies of larger indole-containing molecules, e. g., tryptophan, as well as molecular clusters, such as the investigation of intermolecular interactions in indole-water ${ }^{45[46]}$ or indole-ammonia. [46] In fact, the experiment described here was set up such that the photofragmentation of indole and indole-water clusters could both be measured. Our findings for the photophysics of indolewater ${ }_{1}$ clusters are beyond the scope of this manuscript and will be presented in an upcoming publication. 47,48

\section{Experimental setup}

Figure 1 shows the experimental setup, including a speciesselecting molecular-beam injector. 45[49] A supersonic expansion of a few mbar of indole seeded in 60 bar of helium was provided by a pulsed Even-Lavie valve. ${ }^{[51]}$ The valve was operated at a repetition rate of $250 \mathrm{~Hz}$ and a temperature of $110^{\circ} \mathrm{C}$. The deflector was used to spatially separate different species present in the expansion, including a separation of indole from the helium seed gas.

The molecular beam apparatus was mounted to the CFELASG Multi-Purpose (CAMP) endstation, [52] which was connected to the Petra III synchrotron's variable polarization beamline P04 (53] (circular polarization $>98 \%, 5 \cdot 10^{13}$ photons $/ \mathrm{s}$, 480 bunches, $16 \mathrm{~ns}$ bunch spacing). The molecular beam was crossed by the $420 \mathrm{eV}(\lambda=2.95 \mathrm{~nm})$ synchrotron radiation under an angle of 90 degree inside a double-sided VMIS $[50]$ for si-

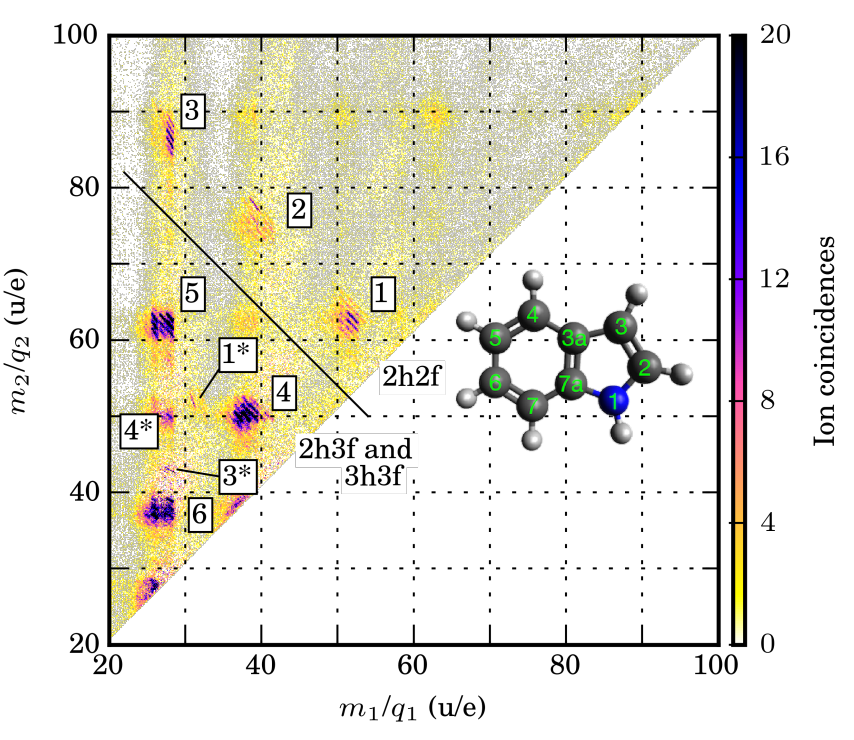

Fig. 2 PEPIPICO spectrum of the first two detected ions of indole following inner-shell ionization. The inset shows the molecular structure of indole with atomic labeling following the IUPAC recommendations. 56 The solid black line is visually separating the $2 \mathrm{~h} 2 \mathrm{f}$ regions from the other regions.

multaneous electron and ion detection. Electrons and ions were detected with a hexanode (electrons) and quadanode (ions) delay line detector (HEX80 and DLD80, RoentDek), respectively. For the data presented, however, the hexanode detector had to be operated as a quadanode due to a defect third delay-line layer. The electronic readout was triggered by the detection of an electron and was set to an acquisition time of $6 \mu \mathrm{s}$, which was long enough to detect ionic fragments with an atomic mass ( $m$ ) -to-charge $(q)$ ratio of up to $\sim 220$. The pulse duration of the molecular beam in the interaction region was about $60 \mu$ sfull width at half maximum (FWHM), resulting in a duty cycle of $\sim 1.5 \%$. A logical gate, synchronized to the arrival time of the molecular beam in the interaction zone, was used to record data in a $200 \mu$ stime window, reducing the absolute number of background events. The overall event rate was on the order of a few hundred events per second. The inset of Figure 1 shows the reconstructed temporal molecular beam profile plus a constant offset due to background events. The background events were used as a background correction in, e. g., Figure 2 In addition to the reconstructed molecular beam profile vertical black lines are shown, indicating the pulse structure of the synchrotron.

\section{Coincidence spectra}

The photofragmentation of indole upon single-photon innershell ionization from the nitrogen and carbon $1 s$ orbitals was investigated via a coincidence measurement between the emitted electrons and the corresponding ionic fragments. A background subtracted PEPIPICO spectrum [54[55] of indole is shown in Figure 2 as a function of the atomic mass-to-charge $m / q$ ratio of the first and second detected ion, $m_{1} / q_{1}$ and $m_{2} / q_{2}$, respectively. The molecular structure of indole is shown in the inset of Figure 2 The PEPIPICO map allows to disentangle different fragmentation channels of indole in the case of at least two detected ionic fragments. Nine principal coincidence regions are observed, which are labeled $1-6,1^{*}, 3^{*}$, and $4^{*}$. A detailed list of the identified fragmentation channels is given in Table 1 
Table 1 Overview of the identified ion-fragmentation channels extracted from the ion coincidence spectrum shown in Figure 2 The indices $i$ and $j$ in the formulas show the number of hydrogen-atom or proton losses that resulted in separate lines with a spacing of $m / q=1 \mathrm{u} / e$ within a given island. Regions 4-6, and $4^{*}$ consist of three heavy neutral/ionic fragments, with numerous different possibilities for hydrogen-atom or proton losses, which are thus not listed explicitly.

\begin{tabular}{|c|c|c|c|c|c|}
\hline Region & Fragmentation type & Fragmentation channel & mass sum (u) & $i$ & $j$ \\
\hline \multirow{2}{*}{1} & \multirow{2}{*}{$2 \mathrm{~h} 2 \mathrm{f}$} & $\mathrm{C}_{4} \mathrm{H}_{4-i}^{+}+\mathrm{C}_{4} \mathrm{NH}_{3-j}^{+}$ & \multirow{2}{*}{$112-117$} & $0-1$ & $0-3$ \\
\hline & & $\mathrm{C}_{3} \mathrm{NH}_{3-i}^{+}+\mathrm{C}_{5} \mathrm{H}_{4-j}^{+}$ & & $0-2$ & $0-3$ \\
\hline $1 *$ & $3 h 2 f$ & $\begin{array}{l}\mathrm{C}_{4} \mathrm{H}_{4}^{+}+\mathrm{C}_{4} \mathrm{~N}^{++} \\
\mathrm{C}_{3} \mathrm{NH}_{2}^{+}+\mathrm{C}_{5} \mathrm{H}_{2}^{++}\end{array}$ & 114 & 0 & 0 \\
\hline \multirow{2}{*}{2} & \multirow{2}{*}{$2 \mathrm{~h} 2 \mathrm{f}$} & $\mathrm{C}_{2} \mathrm{NH}_{3-i}^{+}+\mathrm{C}_{6} \mathrm{H}_{4-j}^{+}$ & \multirow{2}{*}{$112-117$} & $0-3$ & $0-2$ \\
\hline & & $\mathrm{C}_{3} \mathrm{H}_{3-i}^{+}+\mathrm{C}_{5} \mathrm{NH}_{4-j}^{+}$ & & $0-1$ & $0-4$ \\
\hline 3 & $2 \mathrm{~h} 2 \mathrm{f}$ & $\mathrm{CNH}_{2}^{+}+\mathrm{C}_{7} \mathrm{H}_{5-i}^{+}$ & $113-117$ & $0-4$ & 0 \\
\hline $3 *$ & $3 \mathrm{~h} 2 \mathrm{~b}$ & $\mathrm{CNH}_{2}^{+}+\mathrm{C}_{7} \mathrm{H}_{2}^{++}$ & 114 & 0 & 0 \\
\hline 4 & $2 \mathrm{~h} 3 \mathrm{f} / 3 \mathrm{~h} 3 \mathrm{f}$ & $\begin{array}{l}\mathrm{C}_{3} \mathrm{H}_{3}^{+}+\left(\mathrm{C}_{3} \mathrm{NH}_{2}^{+} \text {or } \mathrm{C}_{4} \mathrm{H}_{4}^{+}\right) \\
\mathrm{C}_{2} \mathrm{NH}^{+}+\mathrm{C}_{4} \mathrm{H}_{4}^{+}\end{array}$ & $86-91$ & & \\
\hline $4 *$ & $2 \mathrm{~h} 3 \mathrm{f} / 3 \mathrm{~h} 3 \mathrm{f}$ & $\begin{array}{l}\mathrm{C}_{2} \mathrm{H}_{2}^{+}+\left(\mathrm{C}_{3} \mathrm{NH}_{2}^{+} \text {or } \mathrm{C}_{4} \mathrm{H}_{4}^{+}\right) \\
\mathrm{CNH}_{2}^{+}+\mathrm{C}_{4} \mathrm{H}_{4}^{+}\end{array}$ & $75-79$ & & \\
\hline 5 & $2 \mathrm{~h} 3 \mathrm{f} / 3 \mathrm{~h} 3 \mathrm{f}$ & $\begin{array}{l}\left(\mathrm{C}_{2} \mathrm{H}_{2}^{+} \text {or } \mathrm{CNH}_{2}^{+}\right)+\mathrm{C}_{5} \mathrm{H}_{3}^{+} \\
\mathrm{C}_{2} \mathrm{H}_{2}^{+}+\mathrm{C}_{4} \mathrm{NH}^{+}\end{array}$ & $87-91$ & & \\
\hline 6 & $2 \mathrm{~h} 3 \mathrm{f} / 3 \mathrm{~h} 3 \mathrm{f} / \ldots$ & $\left\{\begin{array}{l}\left(\mathrm{C}_{2} \mathrm{H}_{2}^{+} \text {or } \mathrm{CNH}^{+}\right)+\mathrm{C}_{3} \mathrm{H}_{3}^{+} \\
\mathrm{C}_{2} \mathrm{H}_{2}^{+}+\mathrm{C}_{2} \mathrm{NH}^{+}\end{array}\right.$ & $61-67$ & & \\
\hline
\end{tabular}

The sum of the masses of the fragments in regions 1-3 is equal to the mass of indole, neglecting the loss of hydrogen/protons. Therefore, these fragmentation channels correspond to the generation of two heavy ionic fragments, which are called in the following a two-hole two-fragment (2h2f) fragmentation channel. They are visually separated from the other channels in Figure 2 by the solid black line. Coincidence regions 4-6, and $4^{*}$ are due to fragmentation into three or more fragments, i. e., the total masses of the first two detected ions corresponding to a single event do not add up to the mass of the indole monomer. The missing fragments can be neutral or ionic and the corresponding channels are labeled two-hole three-fragment (2h3f) and three-hole three-fragment (3h3f), respectively. Due to a limited detection efficiency, the $3 \mathrm{~h} 3 \mathrm{f}$ fragments can split into different coincidence regions as, for example, the regions 4 and $4 *$. Both regions have the same 'heavy' second detected ion, i. e., $\mathrm{C}_{3} \mathrm{NH}_{2}^{+}$or $\mathrm{C}_{4} \mathrm{H}_{4}^{+}$, but alternating 'lighter' fragments for the first detected ion. If only the 'lighter' fragments are detected, or if all ions are detected, this fragmentation channel is, in the used representation, part of region 6 . Regions $1^{*}$, and $3 *$ have molecular fragments with the same masses as regions 1 , and 3 , but with different charge distribution, i. e., they contain both, singly and doubly charged ionic fragments and are labeled therefore as three-hole two-fragment ( $3 \mathrm{~h} 2 \mathrm{f}$ ) channels.

If not stated otherwise, the losses of hydrogens or protons will not be considered, and are not included in the labeling of the different fragmentation channels. Further, $2 \mathrm{~h} 2 \mathrm{f}$ and $2 \mathrm{~h} 3 \mathrm{f}$ fragmentation channels are quantified such that they show strong axial recoil, as described in section 4 In contrast, the majority of ions detected in 3h3f fragmentation channels do not show a strong axial recoil. Therefore, if not all ions are detected in a $3 \mathrm{~h} 3 \mathrm{f}$ fragmentation channel, these channels are distinguished from $2 \mathrm{~h} 2 \mathrm{f}$ or $2 \mathrm{~h} 3 \mathrm{f}$ by their axial recoil. Furthermore, due to the stronger Coulomb repulsion between three ionic fragments, the kinetic energy of the $3 \mathrm{~h} 3 \mathrm{f}$ fragments gives a hint toward these fragmentation channels.

Taking this assumptions into account and assuming an ion detection efficiency $\sim 40 \%$, the branching ratios between the main regions of the PEPIPICO spectrum can be estimated to $27 \%$, $51 \%$, and $22 \%$ for $2 \mathrm{~h} 2 \mathrm{f}, 2 \mathrm{~h} 3 \mathrm{f}$ and $3 \mathrm{~h} 2 \mathrm{f} / 3 \mathrm{~h} 3 \mathrm{f}$, respectively. The detection efficiency of the electrons is neglected, leading to an overestimation of the contribution of $3 \mathrm{~h} 2 \mathrm{f}$ and $3 \mathrm{~h} 3 \mathrm{f}$ fragmentation channels. Independent of the electron detection efficiency, the majority of indole molecules is thus fragmenting into three heavy fragments.

If proton and hydrogen transfer processes are neglected, PEPIPICO region 3 and $3 *$ are the only PEPIPICO regions for which the ionic fragments can be uniquely assigned, i.e., $\mathrm{CNH}_{2}+\mathrm{C}_{7} \mathrm{H}_{5-\mathrm{i}}$ corresponding to the atoms $(1,2)$ and $(3,3 \mathrm{a}$, $4,5,6,7,7 \mathrm{a}$ ); see the notation in the inset of Figure 2 . In contrast, PEPIPICO region 1 and 2 consist of a superposition of two fragmentation channels, which can additionally consist of non-unique fragmentation combinations of the indole molecule. Consider, for example, the fragmentation $\mathrm{C}_{3} \mathrm{NH}_{3-\mathrm{i}}+\mathrm{C}_{5} \mathrm{H}_{4-\mathrm{j}}$ of PEPIPICO region 1. The possible atomic combinations for $\mathrm{C}_{3} \mathrm{NH}_{3-\mathrm{i}}$ are $(1,2,3,3 \mathrm{a}),(1,2,3,7 \mathrm{a}),(1,2,7,7 \mathrm{a})$, or $(1,6,7,7 \mathrm{a})$. In the case of $2 \mathrm{~h} 3 \mathrm{f}$ and $3 \mathrm{~h} 3 \mathrm{f}$ fragmentation channels (regions 4-6) the possible combination of ionic fragments is further increased, resulting in an even lower probability to uniquely assigning the fragments. Exceptions are some single coincidence lines within a coincidence region, such as $\mathrm{C}_{4} \mathrm{H}_{4}+\mathrm{C}_{4} \mathrm{NH}_{3}$ (PEPIPICO region 1) whose mass sum is equivalent to the mass of the in dole molecule, i. e., including the mass of all hydrogens. 

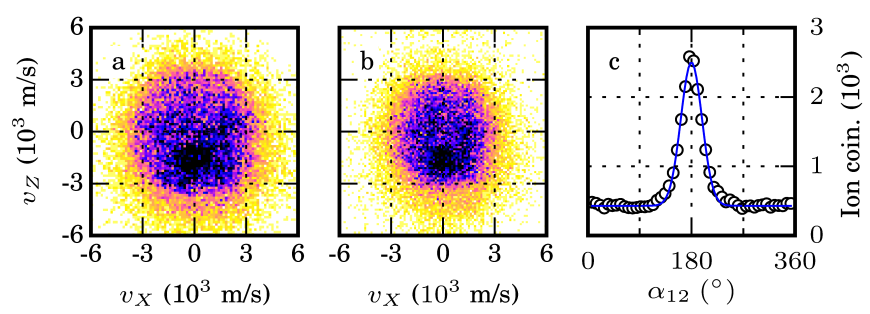

Fig. $3 \mathrm{VMl}$ images of the a) first and b) second detected ion contributing to the $2 \mathrm{~h} 3 \mathrm{f}$ fragmentation channel of coincident region 4. c) Histogram of the angle between the first and the second ion with a Gaussian fit indicated by the blue line.

\section{Fragmentation dynamics}

The VMIS is used to measure the projected velocity vectors of the ionic fragments. Figure 3 a and b show the VMI images for the first and second detected ion in the coincidence region 4. The corresponding fragments are $\mathrm{C}_{3} \mathrm{H}_{3}^{+}$and $\left(\mathrm{C}_{3} \mathrm{NH}_{2}^{+}\right.$or $\left.\mathrm{C}_{4} \mathrm{H}_{4}^{+}\right)$or $\mathrm{C}_{2} \mathrm{NH}^{+}$and $\mathrm{C}_{4} \mathrm{H}_{4}^{+}$; the color scale is the same as Figure 2 The velocity of the VMI was calibrated by the helium-photoelectron recoil for different photon energies ranging from 310 to $420 \mathrm{eV}$. The first detected ions show a slightly higher velocity compared to the second detected ions, which is explained by their smaller mass and the momentum conservation of the fragmenting particles. The increased number of counts visible in the VMI images at $v_{X}=0$ and $v_{Z} \approx-2 \cdot 10^{3} \mathrm{~m} / \mathrm{s}$ is due to background from the carrier gas, which is falsely detected at that corresponding TOF window and does not obey momentum conservation ${ }^{7}$ A histogram of the angular relationship between the first and second detected ions is shown in Figure 3c. The angle $\alpha_{12}$ is defined as counter-clockwise rotation about $Z$ starting from the 2 D velocity vector of the first detected ion. The blue line shows a Gaussian fit centered at an recoil angle of $\alpha_{12}=180^{\circ}$ with a standard deviation (SD) of the recoil angle of $\sigma_{\alpha_{12}}=18.4^{\circ}$. This strong axial recoil between ions in this channel is only observed for a $2 \mathrm{~h} 3 \mathrm{f}$ fragmentation process (vide infra). This is in agreement with the expected fast fragmentation of the molecule due to Coulomb explosion subsequent to inner-shell ionization, and the momentum conservation between the ionic fragments. $\sigma_{\alpha_{12}}$ depends on the fragmentation channel, and is $\sigma_{\alpha_{12}}=12.7^{\circ}$ for the $2 \mathrm{~h} 2 \mathrm{f}$ fragmentation channels, and $\sigma_{\alpha_{12}}=9.8^{\circ}$ and $\sigma_{\alpha_{12}}=9.5^{\circ}$ for the $1^{*}$ and $3 *$ fragmentation channel, which were assigned to a $3 \mathrm{~h} 2 \mathrm{f}$ fragmentation channels. These channels show a stronger confinement in the recoil-frame (RF) because they experience a stronger Coulomb repulsion, which leads to an RF that is more dominated by Coulomb repulsion. In contrast, in a $2 \mathrm{~h} 3 \mathrm{f}$ fragmentation channel the momentum of the Coulomb repulsion is more in competition with the momentum taken up by the heavy neutral fragment, resulting in a less-confined axial recoil.

The angular variations $\sigma_{\alpha_{12}}$ in the recoil-frame can be expressed as a degree of (post-)orientation or alignment in the RF, which is $\left\langle\cos \alpha_{12,2 \mathrm{D}}\right\rangle \approx 0.98,0.99$, and 0.95 , or $\left\langle\cos ^{2} \alpha_{12,2 \mathrm{D}}\right\rangle=$ $0.95,0.97$, and 0.91 , for the $2 \mathrm{~h} 2 \mathrm{f}, 3 \mathrm{~h} 2 \mathrm{f}$, and $2 \mathrm{~h} 3 \mathrm{f}$ fragmenta-

$¥$ These events might be due to a subsequent pulse of the synchrotron radiation ionizing a second particle in the molecular beam within the $6 \mu$ sacquisition time window (Figure 1], which has a small but finite probability. Helium contributes strongest to the signal from the molecular beam and is, therefore, the main background signal.
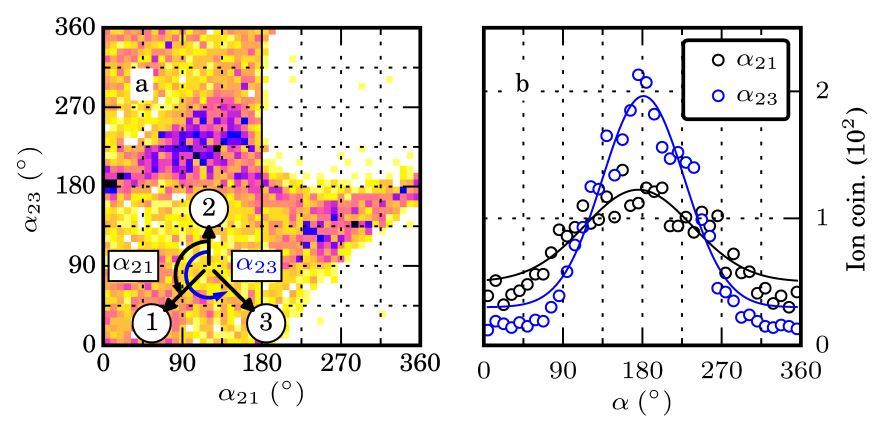

Fig. 4 a) Angular relationship between the ions of the 3 h3f fragmentation of Figure 3. In the right half, only ions that obey momentum conservation are shown. The definition of the angle is indicated by the inset in the top right corner. $\alpha_{21}$ is the angle between the second and first-, $\alpha_{23}$ the angle between the second and third detected ionic fragment. b) Histograms of the angular relationship between the ions of a).

tion channels, respectively. The angular confinement, i. e., the alignment, is comparable to the best laser alignment experiments [57] whereas the directionality, i.e., the orientation, is significantly better. [57|58] Thus, in the case of the planar indole molecule, these RF determinations allow for RF-ARPES of the individual ion fragmentation channels, albeit that the actual angular-resolution quality of the ARPES depend on the specific fragmentation channel.

The deviation in $\sigma_{\alpha_{12}}$ between the $2 \mathrm{~h} 2 \mathrm{f}$ and $2 \mathrm{~h} 3 \mathrm{f}$ can be used to estimate the velocity of the neutral fragment. An explicit assignment of the neutral fragments of PEPIPICO region 4 and 5 is not possible since the neutral fragments cannot be detected. From the tight momentum conservation we infer, however, that the bonds between the neutral and the ionic fragments are broken instantaneously on the timescale of the fragmentation process. In addition, we assume that the missing masses are intact fragments due to the following reasons: First, the ionic fragments dominantly stay intact in the case of a $3 \mathrm{~h} 3 \mathrm{f}$ fragmentation. Second, there is no dominant PEPIPICO region where only a single carbon is missing. Then, in the case of coincidence region 4 a mean velocity of $500 \mathrm{~m} / \mathrm{s}$ can be assigned to a neutral fragment with a mean mass of $27 \mathrm{u}$.

Figure 4a shows the angular correlation between the ions of a 3h3f fragmentation channel; the second and third detected ions have the same masses as the ions shown in Figure 3. i. e., they correspond to the fragments $\mathrm{C}_{3} \mathrm{H}_{3}^{+}$and $\left(\mathrm{C}_{3} \mathrm{NH}_{2}^{+}\right.$or $\left.\mathrm{C}_{4} \mathrm{H}_{4}^{+}\right)$, or $\mathrm{C}_{2} \mathrm{NH}^{+}$and $\mathrm{C}_{4} \mathrm{H}_{4}^{+}$. The first detected ions were previously neutral and are assigned to the ionic fragments $\mathrm{C}_{2} \mathrm{H}_{2}^{+}$or $\mathrm{CNH}^{+}$. The two dimensional histogram shows the angles $\alpha_{23}$ and $\alpha_{21}$ between the $2 \mathrm{D}$ velocity vector of the second-third and secondfirst ion pairs. The definition of the angles with respect to the fragments is visualized by the inset in the top right corner of Figure 4 The angular relationship between these pairs of fragments shows an hourglass-like structure, rotated by approximately $45^{\circ}$. Coincidences outside that structure are due to ions, which do not fulfill momentum conservation. This is illustrated by right part of the same histogram, where only ion combinations are shown that do fulfill momentum conservation to a high degree $(<60 \mathrm{u} \cdot 117 \mathrm{~km} / \mathrm{s})$. Figure $4 \mathrm{~b}$ shows the histogram of the angles $\alpha_{21}$ and $\alpha_{23}$ for ion pairs that obey momentum conservation, and allows therefore for a better comparison of the recoil angle between the $2 \mathrm{~h} 3 \mathrm{f}$ and $3 \mathrm{~h} 3 \mathrm{f}$. These channels have 


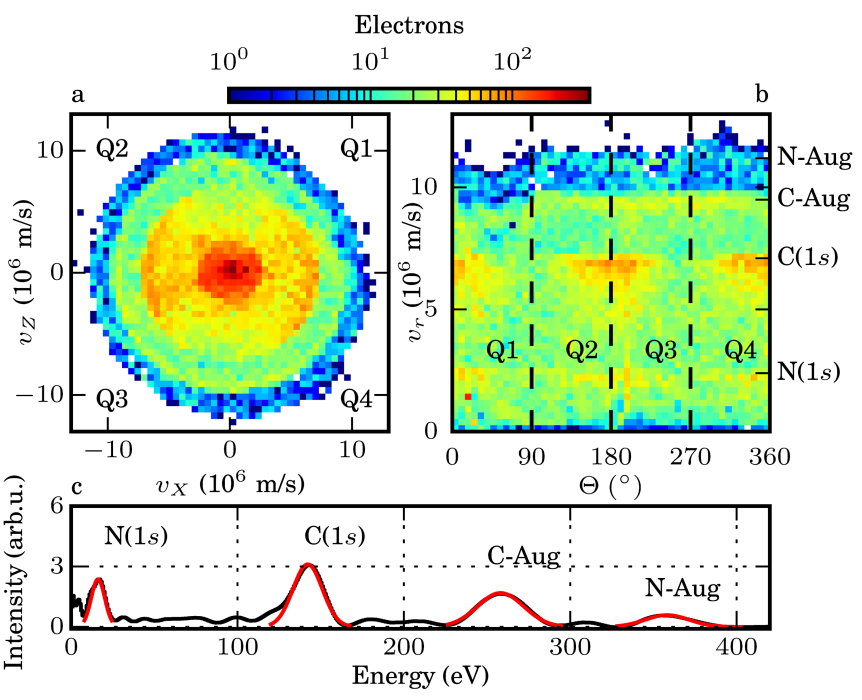

Fig. 5 Photoelectron VMI image of indole in cartesian (a) and polar (b) coordinate systems. Q1-Q4 indicate the four different quadrants of the VMI image. (c) Photoelectron energy spectrum obtained from the inverse-Abel-transformed data of Q2 and Q3 in black. The red curves show Gaussian fits to the assigned electron peaks.

an SD of $\sigma_{\alpha_{21}}=70.3^{\circ}$, and $\sigma_{\alpha_{23}}=50.7^{\circ}$, which is a significantly worse axial recoil compared to the one given in Figure 3 for a $2 \mathrm{~h} 3 \mathrm{f}$ fragmentation channel, and allows therefore to discriminate between both fragmentation channels. This fixed angular relationship between three heavy ionic fragments demonstrates the possibility to reconstruct the three dimensional orientation of the molecule in the laboratory frame provided that the directionality of the moving fragments in the molecular frame are known. Due to the strict planarity of the indole molecule and the immediate Coulomb explosion, the plane of the molecule can be assigned to the recoil plane defined by the three ionic fragments. However, the orientation within the symmetry plane is practically undefined.

\section{Angle-resolved photoelectron spectra}

Figure 5a and b show the electron velocity map in a cartesian and a polar coordinate system, respectively. The photoelectron VMI has been calibrated by photoelectrons originating from single-photon ionization of atomic helium and neon, at photon energies between 310 and $980 \mathrm{eV}$. The labels Q1-Q4 correspond to the four different quadrants of the VMI image; $v_{X}$ and $v_{Z}$ correspond to the electrons velocity component in the laboratory frame, and $v_{r}$ and $\theta$ are the radial and angular coordinate in the polar coordinate system. The electrons were detected in coincidence with PEPIPICO regions $1-5,1^{*}$ and $3^{*}$, with a background correction applied by accepting only events within $2 \sigma$ of the recoil angle of the ions (Figure 3). The 3h3f fragmentation channels of indole have been considered if three ions were detected, if the second and third detected ion were falling into the coincidence regions 4 , and 5 , and if the ions fulfilled momentum conservation (Figure 4b). Region 6 was not used due to a high number of background ions detected in this coincidence region. The electron VMI images of indole show four distinct electron velocities at 2.4, 7.1, 9.5, and $11.2 \cdot 10^{6} \mathrm{~m} / \mathrm{s}$, which correspond to electron energies of 16,143 , 258, and $358 \mathrm{eV}$. The additional slow electrons visible in the center of the VMI image are assigned to background and shake- off electrons from the molecule. The electron energy spectrum, shown in the bottom graph of Figure 5, was obtained by an inverse Abel transformation based on the BASEX algorithm [59] of the second and third quadrant of the electron-VMI image. Quadrants one and four were not used, to avoid the influence of the VMI distortions in these quadrants, which are visible for velocities grater than $\sim 8 \cdot 10^{6} \mathrm{~m} / \mathrm{s}$, and attributed partially to the non-working layer of the hexanode DLD, possible influence of an magnetic field, or a non well-centered interaction region in the VMI. Considering atomic electron binding energies, the nitrogen and carbon $1 \mathrm{~s}$ photoelectron energies would be expected at 10.1 and $135.8 \mathrm{eV}, \underline{60}$ respectively. In pyrrole $\left(\mathrm{C}_{4} \mathrm{H}_{5} \mathrm{~N}\right)$, which corresponds to the five-membered-ring part of indole, the binding energies are chemically shifted and would correspond to photoelectron energies of 14 and $130 \mathrm{eV}$ for nitrogen and carbon $1 s$, respectively. [61] This is a deviation of less than $5 \%$ between the $1 s$ binding energies in pyrrole and indole, which is within the systematic error of our measurement. The observed C KVV-Auger-electron energies agree with the experimentally observed lines in benzene at $243-267 \mathrm{eV}$. ${ }^{[62]}$ The N KVV-Auger-electron energies agree with calculated energies of 356-377 eV. ${ }^{[63]}$ Fitted Gaussians, shown by the red line in Fig. ure 5 c, allow to extract relative intensities of the specific peaks and, thus, ratios of the electron channels. By comparing innershell ionization events, the $\mathrm{N}(1 s)$ and $\mathrm{C}(1 s)$ Gaussian fits show a $26.1 \%$ probability for localized ionization at the nitrogen atom. A similar probability of $24.8 \%$ is obtained by comparing the Auger electron ratio. Both numbers are slightly higher than the expected probability of $16 \%$ by considering the atomic cross sections of $\mathrm{C}$ and $\mathrm{N}$. We attribute this difference to the specific properties of the selected Coulomb explosion channels. The SD of the $\mathrm{N}(1 s)$ and $\mathrm{C}(1 s)$ photolines are $\sigma=4$ and $\sigma=9 \mathrm{eV}$, respectively, which is attributed to the distortions of the VMIS and the low number of electrons of the VMI image. The chemicalshift variations of the different carbon atoms $(\sim 2 \mathrm{eV})$ and the bandwidth of the synchrotron radiation $(0.4 \mathrm{eV})$ are negligible. The anisotropy parameters for the photo- as well as Auger electrons, obtained from the inverse Abel transformation averaged over the FWHM of the photoelectron line, are $\beta_{\mathrm{N}(1 s)}=1.1(0.1)$, $\beta_{\mathrm{C}(1 s)}=1.7(0.1), \beta_{\mathrm{C} \text {-Auger }}=0.2(0.1)$, and $\beta_{\mathrm{N} \text {-Auger }}=0.2(0.1)$. The anisotropy parameter of the Auger electrons is consistent with the expected isotropic distribution of electrons in the laboratory frame. The anisotropy parameter for $\mathrm{C}(1 s)$ photoelectrons is slightly lower and the anisotropy parameter for $\mathrm{N}(1 s)$ photoelectrons is significantly lower than the one, $\beta=2.0$, expected for ionization out of an s-orbital by circularly polarized radiation. We attribute this lowered asymmetry parameters to the interaction of photoelectrons with the potential of the molecule, [64] but also partly to the non-perfect reconstruction.

\section{Electron-ion fragmentation correlation}

The measured coincidences between electrons and ions allow to extract the individual 2D electron VMI spectra of the various ionic fragmentation channels. The $2 \mathrm{~h} 2 \mathrm{f}$ and $2 \mathrm{~h} 3 \mathrm{f}$ ion fragmentation channels show a spectrum similar to the one shown in Figure 5c. The energy spectrum of the $3 \mathrm{~h} 2 \mathrm{f}$ and $3 \mathrm{~h} 3 \mathrm{f}$ fragmentation channels yielded no clear results due to low statistics. Therefore, for the $2 \mathrm{~h} 2 \mathrm{f}, 2 \mathrm{~h} 3 \mathrm{f}, 3 \mathrm{~h} 2 \mathrm{f}$ and $3 \mathrm{~h} 3 \mathrm{f}$ channels, radial velocities of the electrons 2D VMI images, i. e., projected electronvelocity distributions (EVD), for the different ionic channels are 


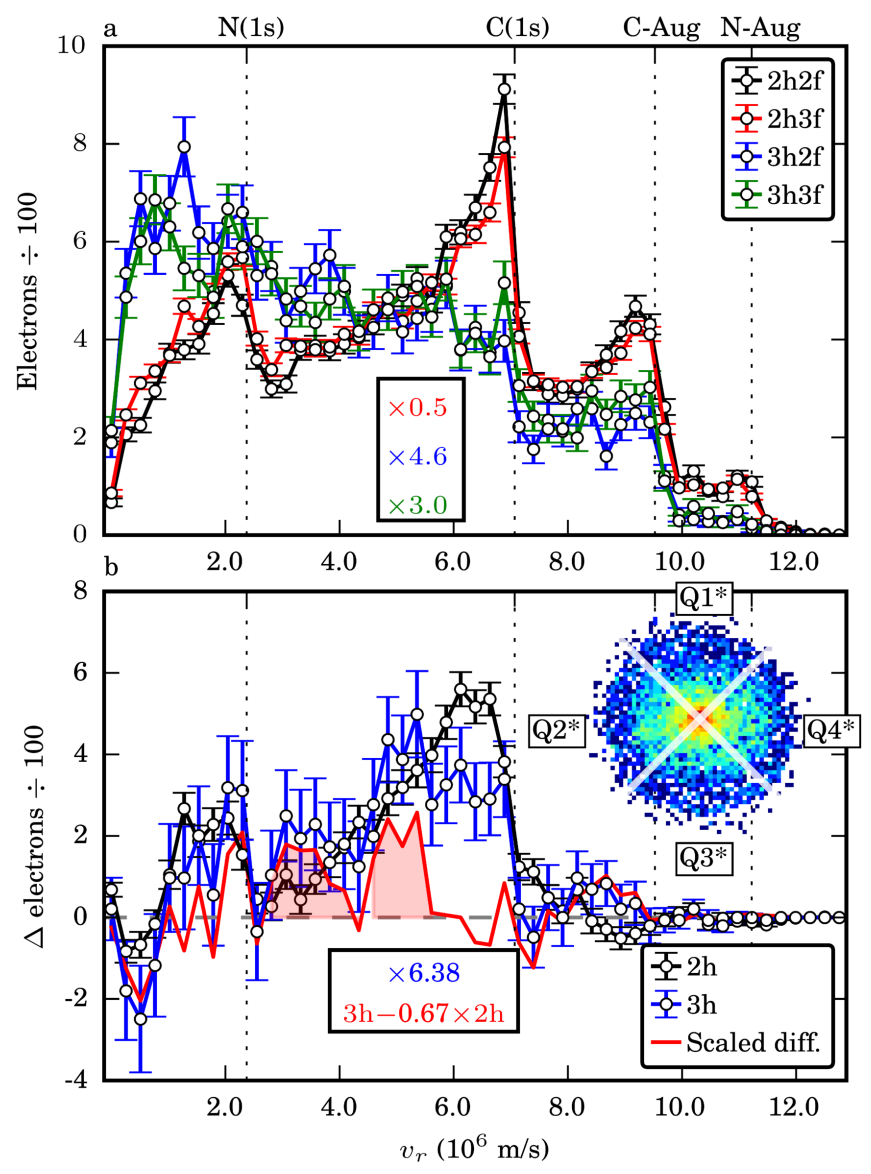

Fig. 6 Radial electron-velocity distributions extracted from the electron VMI. The histograms are normalized to the same number of electrons; the scaling parameters are given in the inset. a) Radial EVD for electrons in coincidence with the ionic fragmentation channels $2 \mathrm{~h} 2 \mathrm{f}, 2 \mathrm{~h} 3 \mathrm{f}$, $3 \mathrm{~h} 2 \mathrm{f}$ and $3 \mathrm{~h} 3 \mathrm{f}$. b) Differential radial plots of the electron VMI retrieved as $\left(\mathrm{Q} 2^{*}+\mathrm{Q} 4^{*}\right)-\left(\mathrm{Q} 1^{*}+\mathrm{Q} 3^{*}\right)$. The labeling of the quadrants is indicated in the inset, which shows the VMI image for electrons detected in coincidence with $3 \mathrm{~h} 2 \mathrm{f}$ and $3 \mathrm{~h} 3 \mathrm{f}$ fragmentation channels.

compared in the following. This time all quadrants of the electron VMI are taken into account. The distortions of the VMI (Figure 5) in quadrant one and four mainly influenced the determined energy for the Auger electrons, which do not have a significant influence on the following discussion.

Figure 6]a shows histograms of the EVD sorted into the contributions of the ion-fragmentation channels $2 \mathrm{~h} 2 \mathrm{f}$ (black), 2h3f (red), 3h2f (blue), and 3h3f (green). The histograms are normalized to the total number of counts; the multiplication factors are given by the inset, and the error bars are given as the statistical error. The connecting lines serve to guide the eye. These electron-velocity distributions clearly group into the two-hole and three-hole channels: The radial EVD for the $2 \mathrm{~h} 2 \mathrm{f}$ and $2 \mathrm{~h} 3 \mathrm{f}$ fragmentation channels (black and red) are very similar. Both show local maxima of electron counts at velocities assigned to the nitrogen and carbon $1 s$ photo- and Auger electrons. The electrons detected between the maxima are due to the projection of the three-dimensional electron velocity distribution onto the two-dimensional detector surface. The $2 \mathrm{~h} 3 \mathrm{f}$ fragmentation channel has the larger contribution of $\mathrm{N}(1 s)$ photoelectrons, whereas the $2 \mathrm{~h} 2 \mathrm{f}$ fragmentation channel has larger contributions from $\mathrm{C}(1 s)$ photoelectrons and their corresponding Auger electrons. This indicates a higher probability for a three-fragment break up if indole is ionized at the nitrogen atom, which can be rationalized by the energy differences between the two possibilities of ionization: Ionization at the $\mathrm{N}(1 s)$ leads to an N KVV-Auger-electron, which results in a mean energy of $46 \mathrm{eV}$ left in the molecule $\$$ whereas ionization at $\mathrm{C}(1 \mathrm{~s})$ leads to a mean energy of $19 \mathrm{eV}$. Thus, it seems the larger energy left in the molecule following $\mathrm{N}(1 s)$ ionization than for $\mathrm{C}(1 s)$ ionization leads to a stronger fragmentation.

The radial EVD for the three-hole fragmentation channels $3 \mathrm{~h} 2 \mathrm{f}$ and $3 \mathrm{~h} 3 \mathrm{f}$, the blue and green lines in Figure 6a, are also similar. In contrast to the $2 \mathrm{~h} 2 \mathrm{f}$ and $2 \mathrm{~h} 3 \mathrm{f}$ radial EVD, the strongest peak of the spectrum is at electron velocities close to the $\mathrm{N}(1 s)$ photoline, and drops-off continuously toward higher electron velocities, with edges at electron velocities corresponding to the carbon $1 s$ photo- and Auger electrons. This overall shift in the electron spectrum toward lower photoelectron energies is attributed partially to a tertiary ionization of indole via electron-impact ionization, and also due to satellite peaks of the photo- and Auger electrons. This is discussed in the second half of the following paragraph based on the angular anisotropy of the electrons.

To extract an angular anisotropy of the electrons radial distribution, the electron VMI is divided into the four quadrants Q1*-Q4* as shown in the inset of Figure 6b; the coordinate system is the same as shown in Figure $5 \mathrm{a}$, but Q1*-Q4* are rotated by $45^{\circ}$ with respect to Q1-Q4. With $\beta$-parameters of 1.1 and 1.7 for the nitrogen and carbon $1 s$ photoelectrons a larger signal is observed in Q2* and Q4* than in Q1* and Q3*. For Auger electrons, which typically show no anisotropy, the same averaged number of counts is expected for all quadrants. The histograms in Figure $6 \mathrm{~b}$ show the radial EVD of the anisotropy $\left(\left(Q 2^{*}+Q 4^{*}\right)-\left(Q 1^{*}+Q 3^{*}\right)\right)$ for electrons detected with two and three ionic fragments in coincidence, i.e., the fragmentation channels $2 \mathrm{~h} 2 \mathrm{f}$ and $2 \mathrm{~h} 3 \mathrm{f}$ are jointly labeled $2 \mathrm{~h}$ (black), and the fragmentation channels $3 \mathrm{~h} 2 \mathrm{f}$ and $3 \mathrm{~h} 3 \mathrm{f}$ are jointly labeled $3 \mathrm{~h}$ (blue). The error bars depict the statistical error, the connecting lines serve to guide the eye, and the histograms are normalized to the number of counts. For the $2 \mathrm{~h}$ fragmentation channels two distinct maxima are visible at electron velocities corresponding to the nitrogen and carbon photoelectrons. The anisotropies of the Auger electrons at $v_{\mathrm{r}} \gtrsim 7 \cdot 10^{6} \mathrm{~m} / \mathrm{s}$ are effectively averaged to zero. The negative values at radial velocities smaller than $1 \cdot 10^{6} \mathrm{~m} / \mathrm{s}$ are attributed to non isotropic noise close to the center of the electron VMI. Comparing the number of electrons assigned to the ionization from nitrogen/carbon shows a probability of approximately $20 \%$ for a localized ionization at the nitrogen atom if the negative values are neglected. This is comparable to the ratio determined from the overall photoelectron intensities in section 5 and, again, slightly higher than expected from the atomic cross sections. The blue histogram, on the other hand, shows electrons in coincidence with the $3 \mathrm{~h}$ fragmentation channels. Here, no clear carbon $1 s$ photoelectron line is visible. Instead, an increased number of electrons is detected at velocities in-between the carbon and nitrogen $1 s$ photoelectron energies. Those electron energies can not be attributed to the earlier determined photo- or Auger electron

$\S$ This energy is determined as the difference between the mean photon energy and the mean summed electron energies, i. e., the sum of photo- and Auger electron energy. 
energies. $\mathrm{N}(1 s)$ photoelectrons do not have enough energy to tertiary ionize indole by electron impact ionization. Also, the contribution from Auger electrons to triply ionize indole can be excluded in this analysis since they do not show an anisotropy in the laboratory frame. Therefore, we attribute those electrons to either inelastically scattered C(1s) photoelectrons and electrons generated by this inelastic scattering through electron impact ionization, or to satellite peaks from the $\mathrm{C}(1 \mathrm{~s})$ photoelectrons. A closer insight is given by the red line in Figure 6 b, which shows a scaled difference between the blue and black spectrum. The scaling was done by a normalization of the number of electrons at $v_{\mathrm{r}}=6.8 \cdot 10^{6} \mathrm{~m} / \mathrm{s}$ to subtract the highest possible contribution from direct photoelectrons. This differencespectrum shows three main areas: the contribution of the nitrogen $1 s$ photoelectrons and two highlighted red areas, which are assigned to those inelastic scattered carbon $1 s$ photoelectrons, electrons emitted upon impact ionization, and satellite peaks from the carbon $1 s$ photoline. These electrons in the red areas have a velocity of $v_{\mathrm{r}}=2.9-4.5 \cdot 10^{6} \mathrm{~m} / \mathrm{s}(24-58 \mathrm{eV})$ and $v_{\mathrm{r}}=4.7-5.7 \cdot 10^{6} \mathrm{~m} / \mathrm{s}(63-92 \mathrm{eV})$. The number of electrons that correspond to these two peaks is about the same, and the sum of the mean electron energy of both peaks is $104 \mathrm{eV}$.

In Figure 6a, the $\mathrm{C}(1 s)$ Auger- and photoelectrons show a similar behavior, i.e., the $2 \mathrm{~h}$ fragmentation channels show a prominent peak, which is absent in the $3 \mathrm{~h}$ fragmentation channels. Therefore, we attribute this change in the radial EVD of Auger electrons also to electron impact ionization or satellite peaks accompanying the Auger electrons.

A quantitative statement about the contribution of the inelastically scattered electrons, electrons from impact ionization, and satellite electrons to the $3 \mathrm{~h} 2 \mathrm{f}$ and $3 \mathrm{~h} 3 \mathrm{f}$ fragmentation channels could, in principle, be extracted from their anisotropy parameter. This was not possible due to the low number of detected electrons. Only for $\mathrm{C}(1 s)$ photoelectrons a lower limit of $43 \%$ can be estimated from Figure 6 b by counting the number of inelastically scattered/satellite electrons (red), which are part of the 3h2f and 3h3f channels (blue).

At the given $\mathrm{C}(1 s)$ photoelectron energy, the atomic cross section for carbon for electron impact ionization and elastic scattering of electrons are both in the order of $200 \cdot 10^{-22} \mathrm{~m}^{2}$. $65 \mid 66$ This implies that elastically-scattered electrons can be detected at comparable signal strengths, e.g., in photoelectron holog-

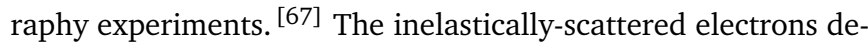
tected here could be separated by an energy-resolving detection scheme, as demonstrated here.

\section{Conclusion}

We have performed a detailed photoionization and photofragmentation study of indole upon single-photon inner-shell ionization at a photon energy of $420 \mathrm{eV}$. This photon energy was chosen such that indole could be locally ionized at its nitrogen atom. Ionization from $\mathrm{C}(1 s)$ was also possible and is the dominant ionization process due to the larger number of carbon atoms present in the molecule. Electrons and ions have been measured in coincidence in a velocity-map-imaging mode to extract $2 \mathrm{D}$ and $3 \mathrm{D}$ velocity vectors of the charged particles.

In the ion-coincidence spectrum of indole, i. e., for the events with more than one ionic fragment observed, indole is fragmenting into two heavy ionic and one neutral fragment in $51 \%$ of the cases. These "heavy" fragments contain, almost exclu- sively, two or more heavier atoms; the loss of hydrogen atoms and protons was also observed, but they were not considered as specific fragments. Fragmentation channels with only two fragments or with three heavy ionic fragments have also been observed and showed contributions of $27 \%$ and $22 \%$, respectively. The PEPIPICO spectrum revealed that the unique assignment of a coincidence region to a carbon atom from a specific position in the molecule is rather the exception than the rule.

The ion-VMI images could be used to reconstruct the recoilframe of the molecules. The fragmentation process was dominated by the Coulomb repulsion of the generated charges. Influence of chemical effects, e. g., the specific potential-energy surfaces, was observed in the recoil frame of the ions for the case of a coexisting heavy neutral fragment. Ion-VMI images of this selected $2 \mathrm{~h} 3 \mathrm{f}$ fragmentation channel were discussed regarding the velocity of the dissociating neutral fragment, showing that the bonds between the neutral and ionic fragments must be broken instantaneously on the timescale of the fragmentation process, i.e., no meta-stable ionic fragments were observed. Fragmentation channels with three ionic fragments also showed a fixed angular relationship. This allowed us, for these channels, to directly determine the alignment of the molecular plane in the laboratory frame. Therefore, the recoilframe and thus, due to the symmetry plane of the molecule, the molecular-frame alignment of the molecular plane in the laboratory frame is uniquely recovered. However, in order to fully reconstruct the three-dimensional alignment and orientation of the indole molecule, i. e., also the orientation inside the molecular plane, the direction of the fragments in this plane would have to be known. This would require elaborate theoretical analysis and is beyond the scope of this paper.

The electron-energy spectrum showed four peaks, which were assigned to photo- and Auger electrons resulting from element-specific ionization at indole's nitrogen as well as carbon atoms. The corresponding asymmetry parameters of these peaks were extracted from an inverse Abel transformation. For the Auger electrons they were isotropic in the laboratory frame, as expected. For the photoelectrons, deviation from the expected asymmetry parameter for photoelectrons from the carbon and nitrogen $1 s$ orbitals have been observed; where "expected" refers to the asymmetry parameter for a single-photon $1 s$ ionization with circularly polarized light. The observed deviation is partly attributed to the interaction of the photoelectrons with the molecular potential, partly due to a non-perfect reconstruction of the asymmetry parameters, as well as deviations due to background signal from slow background and shake-off electrons.

The correlation between ions and electrons showed that different ion fragmentation channels have different electron spectra, i. e., a relationship between the ionization/excitation process, the corresponding electronic states, and the fragmentation process, reflecting the specific potential energy surface. This was shown, for instance, by a comparison of the projected electron energy spectra for the $2 \mathrm{~h} 2 \mathrm{f}$ and $2 \mathrm{~h} 3 \mathrm{f}$ fragmentation channels. In this case it was concluded that inner-shell ionization at the nitrogen edge leads to a higher probability for indole to break up into three heavy fragments.

Evidence for secondary electron-impact ionization as well as satellite photoelectrons was observed in the fragmentation channels where three ionic fragments have been measured. 
Those channels showed less pronounced photolines, primarily observed for the $\mathrm{C}(1 s)$ photoelectrons, as well as signals at electron energies where no photoline is expected. In addition, evidence for satellite peaks of the Auger electrons and inelastically scattered Auger electrons was presented.

Since the cross sections for the observed inelastic scattering and elastic scattering are comparable under the experimental conditions, the possibility of photoelectron-holography experiments is confirmed.

The presented data allowed to record RF-ARPES images of strongly post-oriented indole, albeit that the relation of RF and MF is unknown beyond the common symmetry plane. Due to the low number of events per unique fragmentation channels, i. e., fragmentation channels where specific carbon atoms could be assigned uniquely to the ionic fragment, no statistically significant asymmetries of the electron distribution in the recoilframe were observed.

Overall, our results show that the fragmentation channels depend on the different electronic states, i.e., the chemical potential energy surface, whereas the observed velocities of the fragments are not strongly dependent of these chemical details.

Our work provides the basis for fragmentation studies of larger molecules as well as molecular clusters, such as the indole-derivative tryptophan or indole-water clusters. Comparison of the fragmentation channels and dissociation energies will allow to study the role of solvents on the photophysics of indole upon site specific x-ray ionization. Furthermore, the processes observed here provide information on the indolechromophore-related radiation damage occurring in coherent diffractive imaging of proteins. 68[69]

\section{Acknowledgments}

We acknowledge Evgeny Savelyev for support with the experiment, and Ludger Inhester for fruitful discussions about the photofragmentation of indole. Besides DESY, this work has been supported by the excellence cluster "The Hamburg Center for Ultrafast Imaging - Structure, Dynamics and Control of Matter at the Atomic Scale" of the Deutsche Forschungsgemeinschaft (CUI, DFG-EXC1074); by the Helmholtz Association through the Virtual Institute 419 "Dynamic Pathways in Multidimensional Landscapes", the Helmholtz Young Investigators Program (D.R. and S.B.), and the "Initiative and Networking Fund"; by the European Union's Horizon 2020 research and innovation program under the Marie SkłodowskaCurie Grant Agreement 641789 MEDEA, and by the European Research Council under the European Union's Seventh Framework Programme (FP7/2007-2013) through the Consolidator Grant COMOTION (ERC-Küpper-614507). D.R. also acknowledges support from the U.S. Department of Energy, Office of Science, Basic Energy Sciences, Chemical Sciences, Geosciences, and Biosciences Division (DE-FG02-86ER13491). S.B. also acknowledges support from the Deutsche Forschungsgemeinschaft (B03/SFB755).

\section{References}

1 R. D. Suenram, F. J. Lovas and G. T. Fraser, MicrowaveSpectrum and N-14 Quadrupole Coupling-Constants of Indole, J. Mol. Spectrosc., 1988, 127, 472-480, DOI: 10.1016/0022-2852(88)90135-X

2 W. Caminati and S. Di Bernardo, Microwave spectrum and amino hydrogen location in indole, J. Mol. Struct., 1990, 240, 253, DOI: 10.1016/0022-2860(90)80514-K

3 L. A. Philips and D. H. Levy, The rotationally resolved electronic spectrum of indole in the gas phase, J. Chem. Phys., 1986, 85, 1327-1332, DOI: 10.1063/1.451219.

4 G. Berden, W. L. Meerts and E. Jalviste, Rotationally resolved ultraviolet spectroscopy of indole, indazole, and benzimidazole: Inertial axis reorientation in the $S_{1}\left({ }^{1} L_{b}\right) \leftarrow S_{0}$ transitions, J. Chem. Phys., 1995, 103, 9596-9606, DOI: 10.1063/1.469974.

5 C. Brand, J. Küpper, D. W. Pratt, W. L. Meerts, D. Krügler, J. Tatchen and M. Schmitt, Vibronic coupling in indole: I. Theoretical description of the ${ }^{1} L_{a}-{ }^{1} L_{b}$ interaction and the electronic spectrum, Phys. Chem. Chem. Phys., 2010, 12, 4968, DOI: $10.1039 / \mathrm{c001776k}$.

6 J. Küpper, D. W. Pratt, L. Meerts, C. Brand, J. Tatchen and M. Schmitt, Vibronic coupling in indole: II. experimental investigation of the ${ }^{1} L_{a}-{ }^{1} L_{b}$ interaction using rotationally resolved electronic spectroscopy, Phys. Chem. Chem. Phys., 2010, 12, 4980-4988, DOI: 10.1039/c001778g.

7 T. M. Korter, D. W. Pratt and J. Küpper, Indole- $\mathrm{H}_{2} \mathrm{O}$ in the gas phase. Structures, barriers to internal motion, and $\mathrm{S}_{1} \leftarrow$ $\mathrm{S}_{0}$ transition moment orientation. Solvent reorganization in the electronically excited state, J. Phys. Chem. A, 1998, 102, 7211-7216, DOI: 10.1021/jp982456x

8 C. Kang, T. M. Korter and D. W. Pratt, Experimental measurement of the induced dipole moment of an isolated molecule in its ground and electronically excited states: Indole and indole- $\mathrm{H}_{2} \mathrm{O}$, J. Chem. Phys., 2005, 122, 174301, DOI: $10.1063 / 1.1883635$.

9 J. Hager and S. C. Wallace, Laser Spectroscopy and Photodynamics of Indole and Indole-van der Waals Molecules in a Supersonic Beam, J. Phys. Chem., 1983, 87, 2121-2127, DOI: $10.1021 / \mathrm{j} 100235 \mathrm{a} 019$

10 K. W. Short and P. R. Callis, Evidence of pure ${ }^{1} L_{b}$ fluorescence from redshifted indole-polar solvent complexes in a supersonic jet, J. Chem. Phys., 1998, 108, 10189, DOI: $10.1063 / 1.476478$

11 R. Montero, A. P. Conde, V. Ovejas, F. Castaño and A. Longarte, Ultrafast Photophysics of the Isolated Indole Molecule, J. Phys. Chem. A, 2012, 116, 2698-2703, DOI: 10.1021/jp207750y.

12 R. Livingstone, O. Schalk, A. E. Boguslavskiy, G. Wu, L. Therese Bergendahl, A. Stolow, M. J. Paterson and D. Townsend, Following the excited state relaxation dynamics of indole and 5-hydroxyindole using time-resolved photoelectron spectroscopy, J. Chem. Phys., 2011, 135, 194307, DOI: $10.1063 / 1.3659231$.

13 T. J. Godfrey, H. Yu, M. S. Biddle and S. Ullrich, A wavelength dependent investigation of the indole photophysics via ionization and fragmentation pump-probe spectroscopies, Phys. Chem. Chem. Phys., 2015, 17, 2519725209, DOI: 10.1039/C5CP02975A.

14 H. Stapelfeldt, E. Constant, H. Sakai and P. B. Corkum, Time-resolved Coulomb explosion imaging: A method to measure structure and dynamics of molecular nuclear wave packets, Phys. Rev. A, 1998, 58, 426-433, DOI: 10.1103/PhysRevA.58.426

15 M. Pitzer, M. Kunitski, A. S. Johnson, T. Jahnke, H. Sann, 
F. Sturm, L. P. H. Schmidt, H. Schmidt-Böcking, R. Dörner, J. Stohner, J. Kiedrowski, M. Reggelin, S. Marquardt, A. Schießer, R. Berger and M. S. Schöffler, Direct Determination of Absolute Molecular Stereochemistry in Gas Phase by Coulomb Explosion Imaging, Science, 2013, 341, 10961100, DOI: 10.1126/science.1240362.

16 D. Popova-Gorelova, J. Küpper and R. Santra, Imaging electron dynamics with time- and angle-resolved photoelectron spectroscopy, Phys. Rev. A, 2016, 94, 013412, DOI: 10.1103/PhysRevA.94.013412

17 R. Boll, D. Anielski, C. Bostedt, J. D. Bozek, L. Christensen, R. Coffee, S. De, P. Decleva, S. W. Epp, B. Erk, L. Foucar, F. Krasniqi, J. Küpper, A. Rouzée, B. Rudek, A. Rudenko, S. Schorb, H. Stapelfeldt, M. Stener, S. Stern, S. Techert, S. Trippel, M. J. J. Vrakking, J. Ullrich and D. Rolles, Femtosecond photoelectron diffraction on laseraligned molecules: Towards time-resolved imaging of molecular structure, Phys. Rev. A, 2013, 88, 061402(R), DOI: 10.1103/PhysRevA.88.061402.

18 L. S. Cederbaum, J. Zobeley and F. Tarantelli, Giant Intermolecular Decay and Fragmentation of Clusters, Phys. Rev. Lett., 1997, 79, 4778-4781, DOI: 10.1103/PhysRevLett.79.4778,

19 T. Jahnke, Interatomic and intermolecular Coulombic decay: the coming of age story, J. Phys. B, 2015, 48, 082001, DOI: $10.1088 / 0953-4075 / 48 / 8 / 082001$.

20 J. Zobeley, R. Santra and L. S. Cederbaum, Electronic decay in weakly bound heteroclusters: Energy transfer versus electron transfer, J. Chem. Phys., 2001, 115, 5076-5088, DOI: 10.1063/1.1395555

21 J. Ullrich, R. Moshammer, A. Dorn, R. Dörner, L. P. H. Schmidt and H. Schmidt-Böcking, Recoil-ion and electron momentum spectroscopy: reaction-microscopes, Rep. Prog. Phys., 2003, 66, 1463-1545, DOI: 10.1088/00344885/66/9/203.

22 T. Arion and U. Hergenhahn, Coincidence spectroscopy: Past, present and perspectives, J. Electron. Spectrosc. Relat. Phenom., 2015, 200, 222-231, DOI: 10.1016/j.elspec.2015.06.004.

23 P. Morin, M. Simon, C. Miron, N. Leclercq and D. L. Hansen, Electron-ion spectroscopy: a probe of molecular dynamics, J. Electron. Spectrosc. Relat. Phenom., 1998, 93, 49-60, DOI: 10.1016/S0368-2048(98)00157-1.

24 A. Sugishima, K. Nagaya, H. Iwayama, M. Yao, J. Adachi, Y. Kimura, M. Yamazaki and A. Yagishita, Dissociation dynamics of $\mathrm{C}_{6} \mathrm{H}_{6}$ and $\mathrm{C}_{6} \mathrm{H}_{5} \mathrm{~F}$ molecules following carbon $1 s$ and fluorine $1 s$ photoionization studied by threedimensional momentum imaging method., J. Chem. Phys., 2009, 131, 114309, DOI: 10.1063/1.3224117.

25 R. Boll, A. Rouzée, M. Adolph, D. Anielski, A. Aquila, S. Bari, C. Bomme, C. Bostedt, J. D. Bozek, H. N. Chapman, L. Christensen, R. Coffee, N. Coppola, S. De, P. Decleva, S. W. Epp, B. Erk, F. Filsinger, L. Foucar, T. Gorkhover, L. Gumprecht, A. Hömke, L. Holmegaard, P. Johnsson, J. S. Kienitz, T. Kierspel, F. Krasniqi, K.-U. Kühnel, J. Maurer, M. Messerschmidt, R. Moshammer, N. L. M. Müller, B. Rudek, E. Savelyev, I. Schlichting, C. Schmidt, F. Scholz, S. Schorb, J. Schulz, J. Seltmann, M. Stener, S. Stern, S. Techert, J. Thøgersen, S. Trippel, J. Viefhaus, M. Vrakking, H. Stapelfeldt, J. Küp- per, J. Ullrich, A. Rudenko and D. Rolles, Imaging Molecular Structure through Femtosecond Photoelectron Diffraction on Aligned and Oriented Gas-Phase Molecules, Faraday Disc., 2014, 171, 57-80, DOI: 10.1039/c4fd00037d.

26 B. Wolter, M. G. Pullen, A. T. Le, M. Baudisch, K. DoblhoffDier, A. Senftleben, M. Hemmer, C. D. Schroter, J. Ullrich, T. Pfeifer, R. Moshammer, S. Gräfe, O. Vendrell, C. D. Lin and J. Biegert, Ultrafast electron diffraction imaging of bond breaking in di-ionized acetylene, Science, 2016, 354, 308-312, DOI: 10.1126/science.aah3429.

27 U. Ablikim, C. Bomme, E. Savelyev, H. Xiong, R. Kushawaha, R. Boll, K. Amini, T. Osipov, D. Kilcoyne, A. Rudenko, N. Berrah and D. Rolles, Isomer-dependent fragmentation dynamics of inner-shell photoionized difluoroiodobenzene, Phys. Chem. Chem. Phys., 2017, 19, 13419-13431, DOI: 10.1039/C7CP01379E.

28 B. Erk, D. Rolles, L. Foucar, B. Rudek, S. W. Epp, M. Cryle, C. Bostedt, S. Schorb, J. Bozek, A. Rouzee, A. Hundertmark, T. Marchenko, M. Simon, F. Filsinger, L. Christensen, S. De, S. Trippel, J. Kupper, H. Stapelfeldt, S. Wada, K. Ueda, M. Swiggers, M. Messerschmidt, C. D. Schroter, R. Moshammer, I. Schlichting, J. Ullrich and A. Rudenko, Inner-shell multiple ionization of polyatomic molecules with an intense $\mathrm{x}$-ray free-electron laser studied by coincident ion momentum imaging, J. Phys. B, 2013, 46, 164031, DOI: 10.1088/0953-4075/46/16/164031.

29 C. Bomme, R. Guillemin, T. Marin, L. Journel, T. Marchenko, D. Dowek, N. Trcera, B. Pilette, A. Avila, H. Ringuenet, R. K. Kushawaha and M. Simon, Double momentum spectrometer for ion-electron vector correlations in dissociative photoionization., Rev. Sci. Instrum., 2013, 84, 103104, DOI: 10.1063/1.4824194.

30 E. Kukk, D. T. Ha, Y. Wang, D. G. Piekarski, S. Diaz-Tendero, K. Kooser, E. Itala, H. Levola, M. Alcami, E. Rachlew and F. Martín, Internal energy dependence in x-ray-induced molecular fragmentation: An experimental and theoretical study of thiophene, Phys. Rev. A, 2015, 91, 043417, DOI: 10.1103/PhysRevA.91.043417

31 H. Levola, E. Itala, K. Schlesier, K. Kooser, S. Laine, J. Laksman, D. T. Ha, E. Rachlew, M. Tarkanovskaja, K. Tanzer and E. Kukk, Ionization-site effects on the photofragmentation of chloro- and bromoacetic acid molecules, Phys. Rev. A, 2015, 92, 063409, DOI: 10.1103/PhysRevA.92.063409.

32 D. T. Ha, M. A. Huels, M. Huttula, S. Urpelainen and E. Kukk, Experimental and $<\mathrm{i}>\mathrm{ab}$ initio $</ \mathrm{i}>$ study of the photofragmentation of DNA and RNA sugars, Phys. Rev. A, 2011, 84, 033419, DOI: 10.1103/PhysRevA.84.033419.

33 D. T. Ha, Y. Wang, M. Alcami, E. Itaelae, K. Kooser, S. Urpeainen, M. A. Huels, E. Kukk and F. Martín, Fragmentation Dynamics of Doubly Charged Methionine Phase, J. Phys. Chem. A, 2014, 118, 1374-1383, DOI: 10.1021/jp4113238.

34 E. Shigemasa, J. Adachi, M. Oura and A. Yagishita, Angular Distributions of $1 s \sigma$ Photoelectrons from Fixed-in-Space $\mathrm{N}_{2}$ Molecules, Phys. Rev. Lett., 1995, 74, 359-362, DOI: 10.1103/PhysRevLett.74.359.

35 D. Toffoli, R. R. Lucchese, M. Lebech, J. C. Houver and D. Dowek, Molecular frame and recoil frame photoelectron angular distributions from dissociative photoioniza- 
tion of NO2, J. Chem. Phys., 2007, 126, 054307, DOI: 10.1063/1.2432124

36 D. Dowek, A. Haouas, R. Guillemin, C. Elkharrat, J. C. Houver, W. B. Li, F. Catoire, L. Journel, M. Simon and R. R. Lucchese, Recoil frame photoemission in inner-shell photoionization of small polyatomic molecules, Eur. Phys. J. Special Topics, 2009, 169, 85-93, DOI: 10.1140/epjst/e200900977-8.

37 H. Sann, T. Havermeier, C. Müller, H. K. Kim, F. Trinter, M. Waitz, J. Voigtsberger, F. Sturm, T. Bauer, R. Wallauer, D. Schneider, M. Weller, C. Goihl, J. Tross, K. Cole, J. Wu, M. S. Schöffler, H. Schmidt-Böcking, T. Jahnke, M. Simon and R. Dörner, Imaging the Temporal Evolution of Molecular Orbitals during Ultrafast Dissociation., Phys. Rev. Lett., 2016, 117, 243002, DOI: 10.1103/PhysRevLett.117.243002

38 R. Guillemin, P. Decleva, M. Stener, C. Bomme, T. Marin, L. Journel, T. Marchenko, R. K. Kushawaha, K. Jänkälä, N. Trcera, K. P. Bowen, D. W. Lindle, M. N. Piancastelli and M. Simon, Selecting core-hole localization or delocalization in $\mathrm{CS}_{2}$ by photofragmentation dynamics., Nat. Commun., 2015, 6, 6166, DOI: 10.1038/ncomms7166.

39 A. Landers, T. Weber, I. Ali, A. Cassimi, M. Hattass, O. Jagutzki, A. Nauert, T. Osipov, A. Staudte, M. H. Prior, H. Schmidt-Böcking, C. L. Cocke and R. Dörner, Photoelectron diffraction mapping: Molecules illuminated from within, Phys. Rev. Lett., 2001, 87, 013002, DOI: 10.1103/PhysRevLett.87.013002.

40 T. J. A. Wolf, R. H. Myhre, J. P. Cryan, S. Coriani, R. J. Squibb, A. Battistoni, N. Berrah, C. Bostedt, P. Bucksbaum, G. Coslovich, R. Feifel, K. J. Gaffney, J. Grilj, T. J. Martinez, S. Miyabe, S. P. Moeller, M. Mucke, A. Natan, R. Obaid, T. Osipov, O. Plekan, S. Wang, H. Koch and M. Gühr, Probing ultrafast $\pi \pi^{*} / \mathrm{n} \pi^{*}$ internal conversion in organic chromophores via K-edge resonant absorption, Nat. Commun., 2017, 8, 29, DOI: 10.1038/s41467-017-00069-7.

41 B. K. McFarland, J. P. Farrell, S. Miyabe, F. Tarantelli, A. Aguilar, N. Berrah, C. Bostedt, J. D. Bozek, P. H. Bucksbaum, J. C. Castagna, R. N. Coffee, J. P. Cryan, L. Fang, R. Feifel, K. J. Gaffney, J. M. Glownia, T. J. Martinez, M. Mucke, B. Murphy, A. Natan, T. Osipov, V. S. Petrovic, S. Schorb, T. Schultz, L. S. Spector, M. Swiggers, I. Tenney, S. Wang, J. L. White, W. White and M. Gühr, Ultrafast X-ray Auger probing of photoexcited molecular dynamics., Nat. Commun., 2014, 5, 4235, DOI: 10.1038/ncomms5235.

42 W. Eberhardt, T. K. Sham, R. Carr, S. Krummacher, M. Strongin, S. L. Weng and D. Wesner, Site-Specific Fragmentation of Small Molecules Following Soft-X-Ray Excitation, Phys. Rev. Lett., 1983, 50, 1038-1041, DOI: 10.1103/PhysRevLett.50.1038.

43 Elettra Web Page, 2017, https://vuo.elettra.eu/ services/elements/WebElements.html, Atomic Calculation of Photoionization Cross-Sections and Asymmetry Parameters.

44 A. T. J. B. Eppink and D. H. Parker, Velocity map imaging of ions and electrons using electrostatic lenses: Application in photoelectron and photofragment ion imaging of molecular oxygen, Rev. Sci. Instrum., 1997, 68, 3477-3484, DOI: 10.1063/1.1148310.
45 S. Trippel, Y.-P. Chang, S. Stern, T. Mullins, L. Holmegaard and J. Küpper, Spatial separation of state- and size-selected neutral clusters, Phys. Rev. A, 2012, 86, 033202, DOI: 10.1103/PhysRevA.86.033202

46 A. L. Sobolewski, W. Domcke, C. Dedonder-Lardeux and C. Jouvet, Excited-state hydrogen detachment and hydrogen transfer driven by repulsive ${ }^{1} \pi \sigma^{*}$ states: A new paradigm for nonradiative decay in aromatic biomolecules, Phys. Chem. Chem. Phys., 2002, 4, 1093-1100.

47 T. Kierspel, Dissertation, Universität Hamburg, Hamburg, Germany, 2016, DOI: 10.3204/PUBDB-2017-02127.

48 T. Kierspel, S. Trippel, J. Küpper et al., In preparation.

49 Y.-P. Chang, D. A. Horke, S. Trippel and J. Küpper, Spatially-controlled complex molecules and their applications, Int. Rev. Phys. Chem., 2015, 34, 557-590, DOI: 10.1080/0144235X.2015.1077838

50 C. Bomme et al., In preparation.

51 U. Even, J. Jortner, D. Noy, N. Lavie and N. Cossart-Magos, Cooling of large molecules below $1 \mathrm{~K}$ and He clusters formation, J. Chem. Phys., 2000, 112, 8068-8071, DOI: 10.1063/1.481405.

52 L. Strüder, S. Epp, D. Rolles, R. Hartmann, P. Holl, G. Lutz, H. Soltau, R. Eckart, C. Reich, K. Heinzinger, C. Thamm, A. Rudenko, F. Krasniqi, K. Kühnel, C. Bauer, C.-D. Schroeter, R. Moshammer, S. Techert, D. Miessner, M. Porro, O. Haelker, N. Meidinger, N. Kimmel, R. Andritschke, F. Schopper, G. Weidenspointner, A. Ziegler, D. Pietschner, S. Herrmann, U. Pietsch, A. Walenta, W. Leitenberger, C. Bostedt, T. Moeller, D. Rupp, M. Adolph, H. Graafsma, H. Hirsemann, K. Gaertner, R. Richter, L. Foucar, R. L. Shoeman, I. Schlichting and J. Ullrich, Large-format, high-speed, $\mathrm{X}$-ray pnCCDs combined with electron and ion imaging spectrometers in a multipurpose chamber for experiments at 4th generation light sources, Nucl. Instrum. Meth. A, 2010, 614, 483-496, DOI: 10.1016/j.nima.2009.12.053.

53 J. Viefhaus, F. Scholz, S. Deinert, L. Glaser, M. Ilchen, J. Seltmann, P. Walter and F. Siewert, The Variable Polarization XUV Beamline P04 at PETRA III: Optics, mechanics and their performance, Nucl. Instrum. Meth. A, 2013, 710, 151154, DOI: 10.1016/j.nima.2012.10.110.

54 J. Eland, F. S. Wort and R. N. Royds, A Photoelectron Ion Ion Triple Coincidence Technique for the Study of Double Photoionization and Its Consequences, J. Electron. Spectrosc. Relat. Phenom., 1986, 41, 297-309, DOI: 10.1016/03682048(86)85010-1.

55 L. J. Frasinski, M. Stankiewicz, K. J. Randall, P. A. Hatherly and K. Codling, Dissociative Photoionization of Molecules Probed by Triple Coincidence - Double Time-ofFlight Techniques, J. Phys. B, 1986, 19, L819-L824, DOI: 10.1088/0022-3700/19/23/002.

56 G. P. Moss, Nomenclature of fused and bridged fused ring systems (IUPAC Recommendations 1998), Pure Appl. Chem., 1998, 70, 143-216, DOI: 10.1351/pac199870010143.

57 L. Holmegaard, J. H. Nielsen, I. Nevo, H. Stapelfeldt, F. Filsinger, J. Küpper and G. Meijer, Laser-induced alignment and orientation of quantum-state-selected large molecules, Phys. Rev. Lett., 2009, 102, 023001, DOI: 10.1103/PhysRevLett.102.023001.

58 S. Trippel, T. Mullins, N. L. M. Müller, J. S. Kienitz, 
R. González-Férez and J. Küpper, Two-state wave packet for strong field-free molecular orientation, Phys. Rev. Lett., 2015, 114, 103003, DOI: 10.1103/PhysRevLett.114.103003.

59 V. Dribinski, A. Ossadtchi, V. A. Mandelshtam and H. Reisler, Reconstruction of Abel-transformable images: The Gaussian basis-set expansion Abel transform method, Rev. Sci. Instrum., 2002, 73, 2634, DOI: 10.1063/1.1482156.

60 A. Thompson, I. Lindau, D. Attwood, Y. Liu, E. Gullikson, P. Pianetta, M. Howells, A. Robinson, K.-J. Kim, J. Scofield, J. Kirz, J. Underwood, J. Kortright, G. Williams and H. Winick, X-Ray Data Booklet, Center for X-Ray Optics and Advanced Light Source, Lawrence Berkeley National Laboratory, Berkeley, CA, 2009.

61 S. A. Chambers and T. D. Thomas, Satellite Structure in XRay Photoelectron-Spectra of Gaseous Furan, Pyrrole, and Thiophene, J. Chem. Phys., 1977, 67, 2596-2603, DOI: $10.1063 / 1.435200$

62 F. Tarantelli, A. Sgamellotti, L. S. Cederbaum and J. Schirmer, Theoretical Investigation of Many Dicationic States and the Auger Spectrum of Benzene, J. Chem. Phys., 1987, 86, 2201-2206, DOI: 10.1063/1.452118.

63 M. Thompson, P. A. Hewitt and D. S. Wooliscroft, Analytical aspects of Auger electron spectrometry of gases, Anal. Chem., 1976, 48, 1336-1340, DOI: 10.1021/ac50003a020.

64 P. W. Langhoff, J. C. Arce, J. A. Sheehy, O. Hemmers,
H. Wang, P. Focke, I. A. Sellin and D. W. Lindle, On the angular distributions of electrons photoejected from fixed-in-space and randomly oriented molecules, J. Electron. Spectrosc. Relat. Phenom., 2001, 114-116, 23-32, DOI: 10.1016/S0368-2048(00)00418-7.

65 Y.-K. Kim and J.-P. Desclaux, Ionization of carbon, nitrogen, and oxygen by electron impact, Phys. Rev. A, 2002, 66, 1279, DOI: 10.1103/PhysRevA.66.012708.

66 A. Jablonski, F. Salvat, C. J. Powell and A. Y. Lee, NIST Electron Elastic-Scattering Cross-Section Database Version 4.0, NIST Standard Reference Database Number 64, National Institute of Standards and Technology, Gaithersburg MD, 20899, 2016.

67 F. Krasniqi, B. Najjari, L. Strüder, D. Rolles, A. Voitkiv and J. Ullrich, Imaging molecules from within: Ultrafast angström-scale structure determination of molecules via photoelectron holography using free-electron lasers, Phys. Rev. A, 2010, 81, 033411, DOI: 10.1103/PhysRevA.81.033411

68 R. Neutze, R. Wouts, D. van der Spoel, E. Weckert and J. Hajdu, Potential for biomolecular imaging with femtosecond X-ray pulses, Nature, 2000, 406, 752-757, DOI: $10.1038 / 35021099$.

69 A. Barty, J. Küpper and H. N. Chapman, Molecular Imaging Using X-Ray Free-Electron Lasers, Annu. Rev. Phys. Chem., 2013, 64, 415-435, DOI: 10.1146/annurev-physchem032511-143708. 